\title{
Despliegue y capacidades sanitarias en la región oeste de Afganistán (provincia de Badghis y Herat) de agosto a noviembre 2012
}

\author{
Navarro Suay R. ${ }^{1}$, Rodrigo Arrastio C ${ }^{2}$., Tamburri Bariain R. ${ }^{3}$, López Soberón E. ${ }^{4}$, Pantojo Trancoso \\ C. ${ }^{5}$, Aceituno Jordán P. ${ }^{6}$
}

Sanid. mil. 2013; 69 (1): 48-60; ISSN: 1887-8571

\begin{abstract}
RESUMEN
En la actualidad despliegan en la misión de la Fuerza Internacional de Asistencia a la Seguridad (ISAF) aproximadamente 1500 militares españoles y 40 Guardias Civiles. Este contingente se distribuye principalmente en las localidades de Qala i Now, Herat, Ludina, Muqur y Kabul. La sanidad militar española está presente en estos destacamentos, sus miembros están cumpliendo su misión y se están obteniendo importantes experiencias sobre el terreno. El objetivo del presente estudio es describir cuál es el personal sanitario desplegado, su ubicación, las capacidades materiales de cada escalón, su función y las lecciones identificadas por los autores durante sus estancias en la zona de operaciones de Afganistán.
\end{abstract}

PALABRAS CLAVE: Escalón médico, ISAF, Afganistán.

Deployment and medical capabilities in the west region of Afghanistan (Badghis and Herat provinces) from august to november 2012 SUMMARY: Actually, in International Security Assistance Force (ISAF) are deployed approximately 1500 Spanish soldiers and 40 Guardia Civil members. This force is located in Qala I Now, Herat, Ludina, Muqur and Kabul. The Spanish medical Corp is in these camps too, their members are carry out the mission and they are learning important medical experiences in the field. The objective is to show which is the medical personnel deployed, position, material capability in each echelon, function and the lessons identified by authors during their deployment in Afghanistan.

KEY WORDS: Medical echelon, ISAF, Afghanistan.

\section{INTRODUCCIÓN}

Las tropas españolas que están desplegadas en Afganistán contribuyen, junto a efectivos de otros 49 países, a la misión de la Fuerza Internacional de Asistencia a la Seguridad (ISAF). Las unidades españolas participan en esta misión desde 2002, inicialmente en Kabul y desde 2004, encuadradas en el Mando Regional Oeste (RC-W), desempeñando cometidos relacionados con la seguridad, la formación y mentorización de las fuerzas de seguridad afganas. Además, España contribuye con personal en los cuarteles generales multinacionales y desarrolla las tareas inherentes al propio sostenimiento logístico. En la actualidad despliegan en ISAF, aproximadamente 1500 militares españoles y 40 Guardias Civiles. Este contingente se distribuye principalmente en las localidades de Qala i Now, Ludina, Muqur (provincia de Badghis), Herat y Kabul ${ }^{1}$.

${ }^{1}$ Cap. Médico. Hospital Central de la Defensa «Gómez Ulla». Servicio de Anestesiología y Reanimación. Madrid. España.

2 Cap. Médico. Unidad Médica de Apoyo Aéreo al Despliegue. Zaragoza. España.

3 Cap. Médico. Hospital Central de la Defensa «Gómez Ulla». Servicio de Traumatología. Madrid. España.

${ }^{4}$ Cap. Médico. Hospital Central de la Defensa «Gómez Ulla». Servicio de Cardiología. Madrid. España.

${ }^{5}$ Cap. Psicólogo. Clínica militar de Ceuta. España.

${ }^{6}$ Cap. Enfermero. III Bandera Paracaidista «Ortíz de Zárate». Murcia. España.

Dirección para correspondencia: Ricardo Navarro Suay. Servicio de Anestesiología y Reanimación. Hospital Central de la Defensa «Gómez Ulla». Glorieta del Ejército s/n. CP: 27047 Madrid.r navarro suay@yahoo.es

Recibido: 19 de noviembre de 2012

Aceptado: 4 de diciembre de 2012
La sanidad militar española está presente en estos destacamentos (Figura 1), sus miembros están cumpliendo su misión y se están obteniendo importantes experiencias sobre el terreno.

El objetivo del presente estudio es describir cuál es el personal sanitario desplegado, su ubicación, las capacidades materiales de cada escalón sanitario, su función y las lecciones identificadas por los autores durante sus estancias en la zona de operaciones de Afganistán desde agosto a noviembre de 2012.

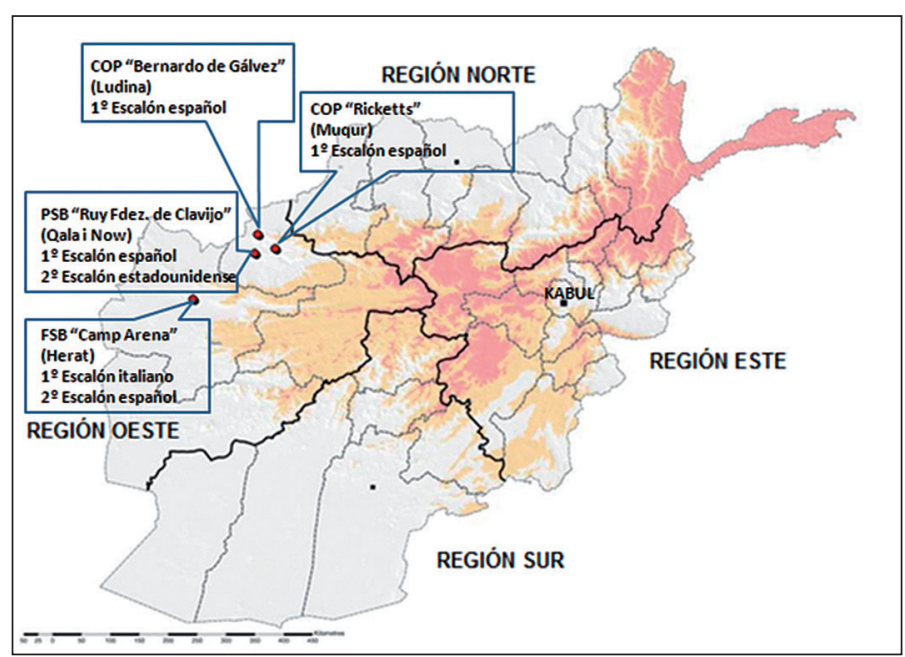

Figura 1. Escalonamiento sanitario aliado de las provincias de Badghis y Herat. COP: Puesto avanzado de combate. PSB: Base de apoyo provincial. FSB: Base de apoyo avanzado. 


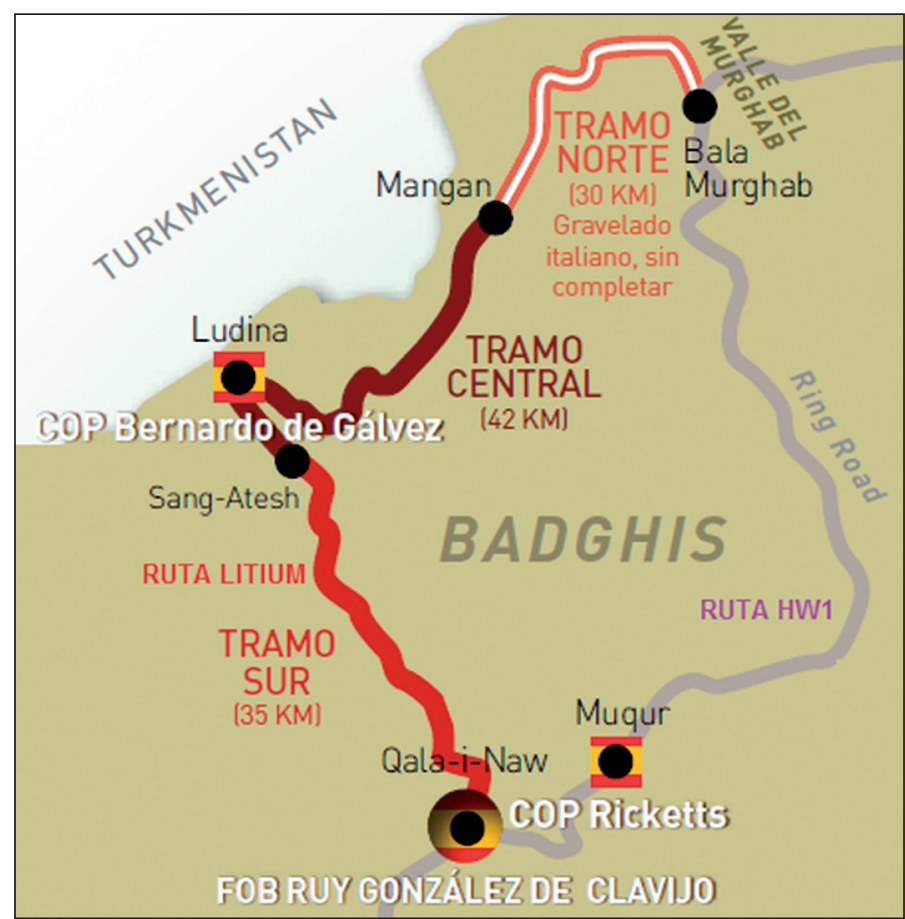

Figura 2. Rutas entre las distintas instalaciones sanitarias. Imagen modificada de Montánchez E. Ruta Lithium, misión cumplida. Revista española de defensa 2012; 287: 15-21.

\section{SITUACIÓN DE LA ZONA DE RESPONSABILIDAD ESPAÑOLA EN LA ACTUALIDAD}

A mediados del año 2008 se planteó un cambio en el centro de gravedad de las operaciones de nuestras fuerzas desplegadas en Afganistán desde la provincia de Herat a la de Badghis. Esta situación supuso un importante incremento de personal y medios desplegados en esta última provincia situada en el norte de la región oeste de Afganistán².

Desde la llegada de las unidades españolas a la provincia de Badghis a mediados del 2005, se ha ido creando una zona de seguridad que se inició en la localidad de Qala i Now para posteriormente extenderse a lo largo de dos rutas principales (la ruta Lithium por el oeste y la HW1 por el este). A medida que el despliegue se extendía por dichas rutas se ocuparon varias posiciones avanzadas de combate (COP) en Muqur (Ricketts), Ludina (Bernardo de Gálvez II) y Darra-i-Bum (Hernán Cortés), esta última ya traspasada al ejército afgano desde principios de $2012^{3}$ (Figura 2).

Durante la realización de este artículo, la principal fuerza española en la provincia de Badghis (ASPFOR XXXI) pertenecía a la brigada paracaidista (1.200 militares). El resto del contingente desplegado en esta provincia procedía del batallón de asuntos civiles 1, el regimiento de inteligencia 1, la agrupación de apoyo logístico 11, controladores aéreos avanzados (ejército del aire e infantería de marina), regimiento Palma 47 (OMLT Muqur), infantería de marina (OMLT Ludina) y una unidad de policía militar de la Guardia Civil ${ }^{4}$. En Herat, España lidera la base de apoyo avanzado. Hay desplegados unos 300 efectivos, la mayor parte del ejército del aire. Además en Kabul, se encuentran aproximadamente 150 militares españoles, la mayor parte

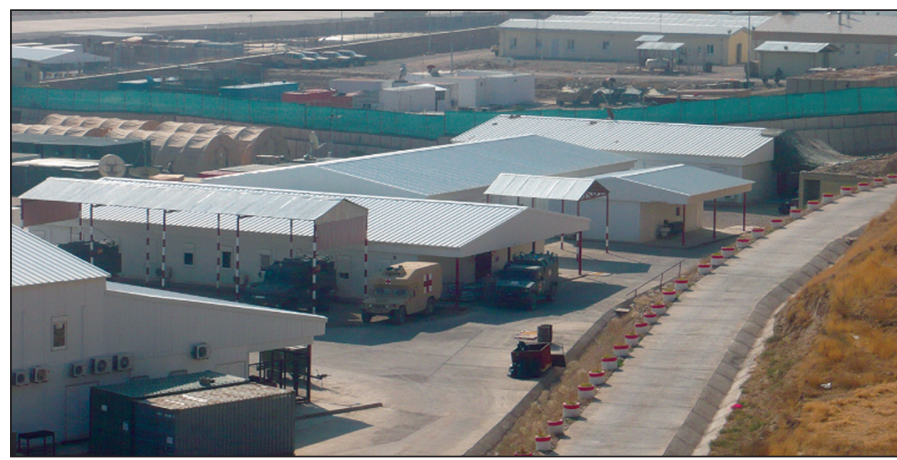

Figura 3. Zona del 1. ${ }^{e r}$ y $2 .^{\circ}$ escalón de la PSB «Ruy González de Clavijo» (Qal i Now) vista aérea. Fotografía del autor.

de ellos destinados en el Cuartel General de Despliegue Rápido de la OTAN (Bétera, Valencia) ${ }^{1}$.

El ministerio de Defensa estima que en el país hay 30.000 insurgentes, de los que 10.000 son talibanes. Calculan que hay entre 250 y 500 talibanes por provincia y una cantidad variable de insurgentes ${ }^{5}$. Los enfrentamientos son frecuentes en la provincia de Badghis (60 durante el primer semestre de 2012 $)$, la insurgencia actúa principalmente con fuego de armas ligeras, lanzagranadas portátil y mediante la colocación de artefactos explosivos improvisados (IED) sobre las rutas de desplazamiento ${ }^{1}$.

\section{Características principales de los destacamentos españoles}

\section{PSB (Base de Apoyo Provincial) "Ruy González de Clavijo" (Qala e Now-Badghis-) (Figura 3)}

La base Ruy González de Clavijo ha sido bautizada así en honor al embajador del rey Enrique III de Castilla, en la corte del gran Tarmerlán en Samarcanda, actual Uzbekistán, a donde llegó en septiembre de 1404.

Fue inaugurada el 13 de julio de 2010. Tiene un perímetro de $6 \mathrm{~km}$ y una superficie de 70 hectáreas. La base está construida en tres áreas escalonadas en altura.

Las instalaciones se estructuran en cuatro zonas. Zona de mando (en ella se encuentran todas las instalaciones relacionadas con esta función), zona de servicios (incluye cocina/comedor, cantina lavandería, cooperativa, biblioteca, locutorio, capilla y peluquería), zona de vida (tanto de personal militar como civil, que tras sucesivas ampliaciones puede albergar hasta 1500 personas) y zona logística (abastecimiento, mantenimiento, transporte incluido el estacionamiento de aeronaves de ala fija y rotatoria y la asistencia sanitaria $)^{2}$. La fuerza en revista es de aproximadamente 600 efectivos.

\section{COP «Ricketts» (Muqur -Badghis-) (Figura 4)}

La COP Ricketts está levantada sobre los restos de un fuerte británico del siglo XIX.

Esta posición avanzada de combate fue ocupada por fuerzas españolas tras la cesión por parte de militares estadounidenses en marzo de 2012. Cuenta con gimnasio, puestos de acceso de internet y telefónicos, cocina, dos comedores, zona de aparca- 


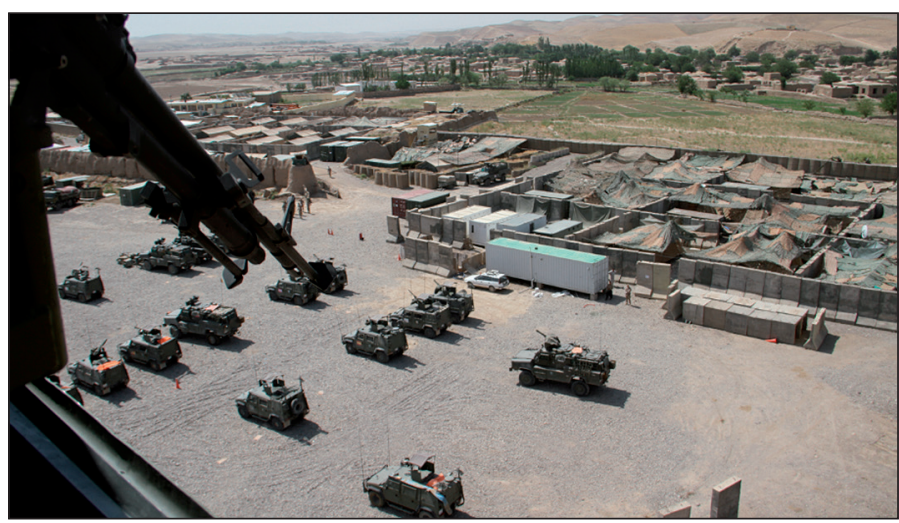

Figura 4. $C O P «$ Rickkets» (Muqur) vista aérea.

miento, primer escalón sanitario y zona de toma de helicópteros $^{3}$. Está separado de Qala i Now $27 \mathrm{~km}^{5}$. La fuerza en revista es de aproximadamente 240 efectivos.

\section{COP «Bernardo de Gálvez II» (Ludina-Badghis-) (Figura 5)}

Esta COP recibió su nombre en recuerdo de Bernardo de Gálvez, militar español que apoyó a fuerzas estadounidenses durante la independencia de este país. Entre sus instalaciones cuenta con cocina, comedor, gimnasio, zona de puestos de acceso a internet y telefónicos, aparcamiento, primer escalón sanitario y zona de toma de helicópteros. Está ubicada a aproximadamente $39 \mathrm{~km}$ de la ciudad de Qala i Now. La fuerza en revista es de aproximadamente 240 efectivos.

\section{FSB (Base de Apoyo Avanzado) "Camp Arena" (Herat)}

(Figura 6)

El día 18 de mayo de 2005 España asumió el mando de la Base de Apoyo Avanzado (Forward Support Base-FSB) de Herat, denominada «Camp Arena». Su misión principal es dar apoyo a los 4 equipos de reconstrucción provincial (PRT) de Farah, Chaghcharan, Herat y Qala-I-Now a cargo de Estados Unidos, Lituania, Italia y España respectivamente. Está localizada junto al aeropuerto civil de Herat a unos $5 \mathrm{~km}$ de

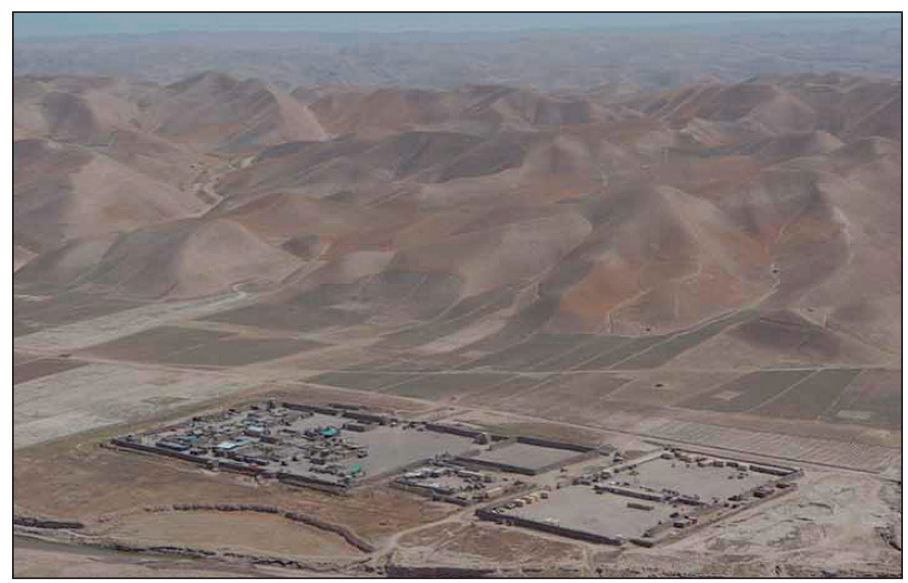

Figura 5. COP «Bernardo de Gálvez II» (Ludina) vista aérea.

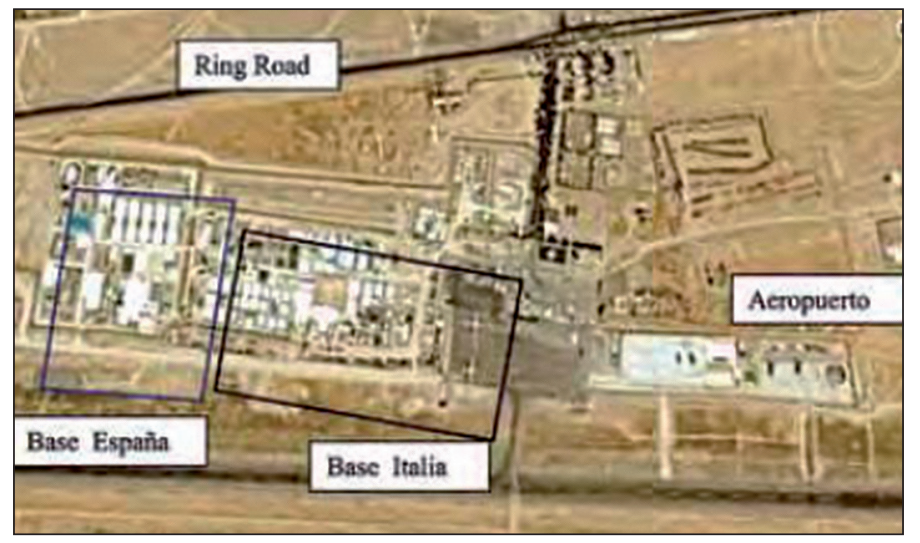

Figura 6. FSB «Camp Arena» (Herat), imagen aérea detallando las principales partes de la misma. Tomada de Maimir F, Sánchez MA, García JM, García RL, Torner A. Brote de gripe A H1N! en la base española de Camp «Arena» (Herat, Afganistán) durante julio y agosto de 2009. Sanid mil. 2011; 67(1): 31-5.

esa ciudad. Está dividida en 4 grandes áreas: zona del CATO (combined air terminal operation) norte, campamento italiano, campamento español y zona del CATO sur. En la FSB están desplegados el Estado Mayor de la FSB, Jefatura de Personal, Unidad de Apoyo Logístico, Unidad de Apoyo a las Operaciones Aéreas, Unidad CIS, $2 .^{\circ}$ escalón sanitario, una compañía HELISAF, compañía ASPUHEL y Mizar. En el recinto de la base se alojan unos 2.000 militares $^{7}$. La distancia entre Qal i Now y Herat es de prácticamente $180 \mathrm{Km}$. La fuerza en revista es de aproximadamente 300 efectivos.

\section{DESPLIEGUE SANITARIO ESPAÑOL EN ZONA DE OPERACIONES}

\section{Personal (Tabla 1)}

Provincia de Badghis (1er escalón de Qal i Now, de Muqur y de Ludina) (Figura 7)

El personal del cuerpo militar de sanidad (CMS) está integrado en zona de operaciones en el ejército de tierra. Los oficiales médicos en territorio nacional están destinados en el ejército de tierra, órgano central, dirección general de reclutamiento y en-

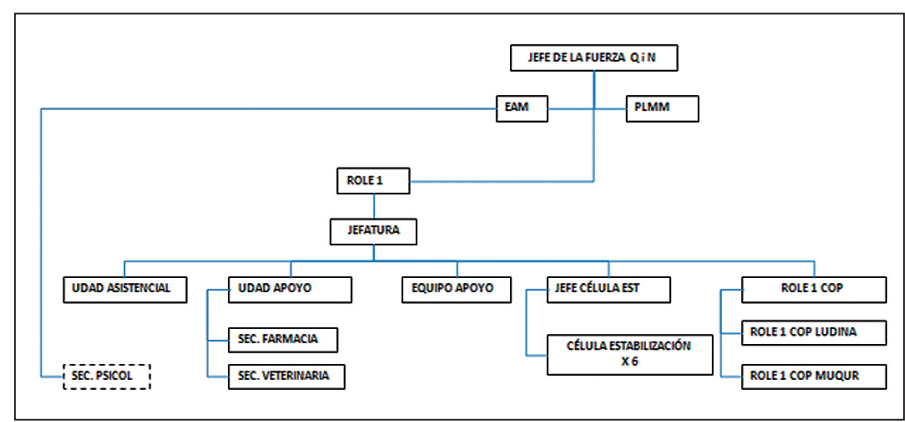

Figura 7. Organigrama de la fuerza en PSB de Qala $i$ Now (específico sanitario). EAM: Equipo de apoyo al mando. PLMM: Plana mayor de mando. UDAD: Unidad. SEC: Sección. EST: Estabilización. COP: Puesto avanzado de combate. 
Tabla 1. Localización, escalonamiento y efectivos sanitarios desplegados en la provincia de Badghis y Herat de la región oeste de Afganistán en noviembre 2012

\begin{tabular}{|c|c|}
\hline Localización & Escalonamiento y efectivos \\
\hline \multirow{4}{*}{$\begin{array}{l}\text { PSB } \\
\text { «Ruy González de Clavijo» } \\
\text { Qal i Now } \\
\text { (Badghis) }\end{array}$} & $\begin{array}{l}\text { 1. er Escalón sanitario español (función Role 1): } \\
\text { Teniente Coronel médico (1) -jefe--, oficial veterinario (1), oficial farmacéutico (1), oficial psicólogo (1), oficial enfermero (1), } \\
\text { suboficial (1), conductor/sanitario de tropa (5). }\end{array}$ \\
\hline & $\begin{array}{l}\text { Células de estabilización: x7 } \\
\text { Oficial médico (1), oficial enfermero (1), conductor/sanitario de tropa (1). }\end{array}$ \\
\hline & $\begin{array}{l}\left.\text { 2. }{ }^{\circ} \text { Escalón sanitario estadounidense (función Role } 2 \mathrm{LM} \approx \mathrm{FST}\right)^{*} \text { : } \\
\text { Oficial médico cirujano general }(1) \text {, oficial médico traumatólogo }(1) \text {, oficial médico anestesiólogo (1), oficial enfermera (1), } \\
\text { sanitario técnico de quirófano (2), sanitario técnico de urgencia (2), oficial de logística (1), suboficial paramédico (1) }\end{array}$ \\
\hline & $\begin{array}{l}\text { Equipo de aeroevacuación estadounidense: X3 } \\
\text { Paramédico (1) }\end{array}$ \\
\hline COP «Rickkets» & 1. er Escalón sanitario español (función Role 1): \\
\hline Muqur (Badghis) & Oficial enfermero (1), conductor/sanitario de tropa (1). \\
\hline COP «Bernardo de Gálvez» & 1. ${ }^{\text {er }}$ Escalón sanitario español (función Role 1): \\
\hline Ludina (Badghis) & Oficial enfermero (1), conductor/sanitario de tropa (1). \\
\hline \multirow[t]{5}{*}{$\begin{array}{l}\text { FSB } \\
\text { «Camp Arena» Herat }\end{array}$} & $\begin{array}{l}\text { 1. er Escalón sanitario italiano (función Role 1): } \\
\text { Oficial médico (1)-jefe-, oficial médico (1), oficial médico reservista voluntario (1), oficial veterinario (2), oficial farmacéutico } \\
\text { (1), oficial psicólogo (1), suboficial enfermero (2), suboficial enfermero reservista voluntario (2). }\end{array}$ \\
\hline & $\begin{array}{l}\left.\text { 2. }{ }^{\circ} \text { Escalón sanitario español (función Role } 2 \mathrm{E}\right) \text { : } \\
\text { Teniente Coronel médico (1) -jefe-, oficial médico -triaje- (1), oficial médico -UCI- (1), oficial médico/farmacéutico } \\
\text {-laboratorio- (1), oficial veterinario (1), oficial farmacéutico (1), oficial psicólogo (1), oficial odontólogo (1), oficial } \\
\text { enfermero (5), suboficial (2), tropa (7). }\end{array}$ \\
\hline & $\begin{array}{l}\text { Apoyo estadounidense al } 2 \text { Escalón sanitario español: } \\
\text { Oficial médico especialista en laboratorio (1), oficial médico radiólogo (1), oficial médico internista (1), oficial médico } \\
\text { anestesiólogo (1), oficial médico cirujano (1), oficial médico traumatológico (1), oficial farmacéutico (1), enfermero (5), } \\
\text { enfermero de quirófano (2), sanitario técnico en farmacia (1), sanitario técnico en radiología (1), paramédicos (3), conductor } \\
\text { (1), sanitario técnico logístico (1), sanitario técnico en comunicaciones (1), sanitario técnico instrumentista (1). }\end{array}$ \\
\hline & $\begin{array}{l}\text { Apoyo búlgaro al } 2 .^{\circ} \text { Escalón sanitario español: } \\
\text { Oficial médico anestesiólogo (2), oficial médico cirujano (4), enfermera de quirófano (4). }\end{array}$ \\
\hline & $\begin{array}{l}\text { Equipo de aeroevacuación médica español: } 2 \text { tripulaciones formadas por Oficial médico (1), oficial enfermero (1), sanitario (1) } \\
\text { Equipo de aeroevacuación italiano: Oficial médico (1), suboficial enfermero (2) }\end{array}$ \\
\hline
\end{tabular}

El personal español desplegado en Kabul, recibe el apoyo sanitario inmediato por parte de países aliados pertenecientes a la OTAN. Dentro del cuartel general español desplegado en Kabul hay un oficial médico comisionado en funciones de logística sanitaria.

A partir de noviembre de 2012 se estima que el equipo búlgaro sea sustituido por un equipo quirúrgico español ${ }^{80}$.

En la FSB "Camp Arena" de Herat, se encuentra el Medical Advisor del RCW (nacionalidad italiana) con función de asesoramiento sanitario al mando.

FST: Foward Surgical Team: Equipo quirúrgico avanzado del ejército estadounidense.

* La composición de un equipo quirúrgico avanzado del ejército estadounidense es doble del que se describe, debido a que la mitad restante está desplegada en la provincia de Farah (Afganistán)

señanza militar o guardia civil. Los oficiales farmacéuticos, veterinarios, psicólogos y enfermeros están destinados únicamente en el ejército de tierra. Asimismo, el suboficial y los sanitarios/ conductores proceden de la unidad militar principal desplegada en zona de operaciones.

Además, el 1. ${ }^{\text {er }}$ escalón de Qal i Now sirve de base principal para 7 células de estabilización (la tripulación de cada una es de: oficial médico, oficial enfermero y sanitario-conductor).

Todo el personal se encuentra en el 1. ${ }^{\text {er }}$ escalón de Qal i Now, excepto las tripulaciones de las células (que frecuentemente se desplazan para realizar la misión encomendada) y los oficiales enfermeros y sanitarios comisionados a las dos COP (Muqur y Ludina $)^{8}$.

\section{Provincia de Herat $\left(2 .^{\circ}\right.$ escalón de Herat)}

En este caso, el personal del cuerpo militar de sanidad está desplegado en zona de operaciones como miembros adscritos al ejército del aire. Los oficiales médicos en España están destinados en el ejército del aire y órgano central. Los oficiales farmacéuticos, veterinarios, psicólogos, odontólogos, enfermeros, suboficiales y tropa también están destinados en el ejército de aire ${ }^{9,10}$.

La tripulación de los dos equipos de aeroevacuación médica está compuesta por oficial médico, oficial enfermero y sanitario. Los oficiales deben haber realizado el curso de médico y enfermero de vuelo respectivamente. Por su parte, el sanitario está destinado en la Unidad Médica de Aeroevacuación. Los oficiales médicos y los sanitarios dependen orgánicamente del $2^{\circ}$ escalón sanitario, mientras que el oficial enfermero pertenece al destacamento de helicópteros (HELISAF) ${ }^{10}$.

La estancia media de los oficiales del CMS desplegados en zona de operaciones es de aproximadamente 80 días $^{11,12}$.

\section{Instalaciones}

Dentro de la PSB «Ruy González de Clavijo» de Qal i Now se encuentra el primer y segundo escalón sanitario compartiendo las mismas instalaciones (Figura 8 y 9). El primero es español y el segundo es estadounidense. Cuenta con las siguientes instalaciones fijas: sala de triaje, sala de curas, quirófano, sala de 
reanimación, sala de hospitalización, sala de rayos $\mathrm{X}$, consulta médica, farmacia, planta de oxígeno, despacho de veterinaria, almacenes de farmacia, veterinaria y de células de estabilización y zona de vida.

Las instalaciones sanitarias se encuentran a apenas 200 metros de la zona de toma de helicóptero y a unos 500 metros de la pista de aterrizaje. Para transportar las bajas dentro de la base, se dispone de una ambulancia blindada Lince (española), un Hummer ambulancia y una mula mecánica (estadounidense).

En la COP «Ricketts» de Muqur (Figura 10) se dispone de un primer escalón sanitario con consulta, zona de estabilización y un despacho médico. El área de vida está separada de la anterior. Las instalaciones no son permanentes. Esta COP cuenta en su interior con una superficie de toma de helicópteros, que dista del escalón sanitario aproximadamente 200 metros. No se cuenta con ninguna ambulancia.

La COP «Bernardo de Gálvez II» de Ludina (Figura 11) cuenta con otro primer escalón sanitario. Únicamente dispone de una tienda de campaña con área de consulta y estabilización que comparte superficie con la zona de vida del personal sanitario. El área de toma de helicóptero está situada en las inmediaciones de la COP. Tampoco cuenta con ambulancia.

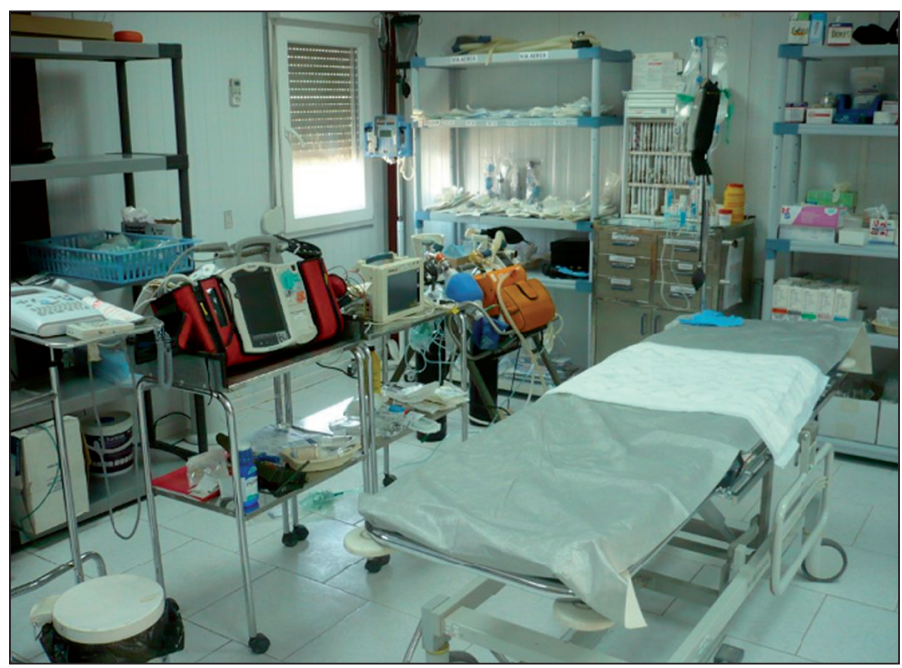

Figura 8. $1 .^{e r}$ Escalón sanitario PSB «Ruy González de Clavijo» (Qala i Now). Fotografía del autor.

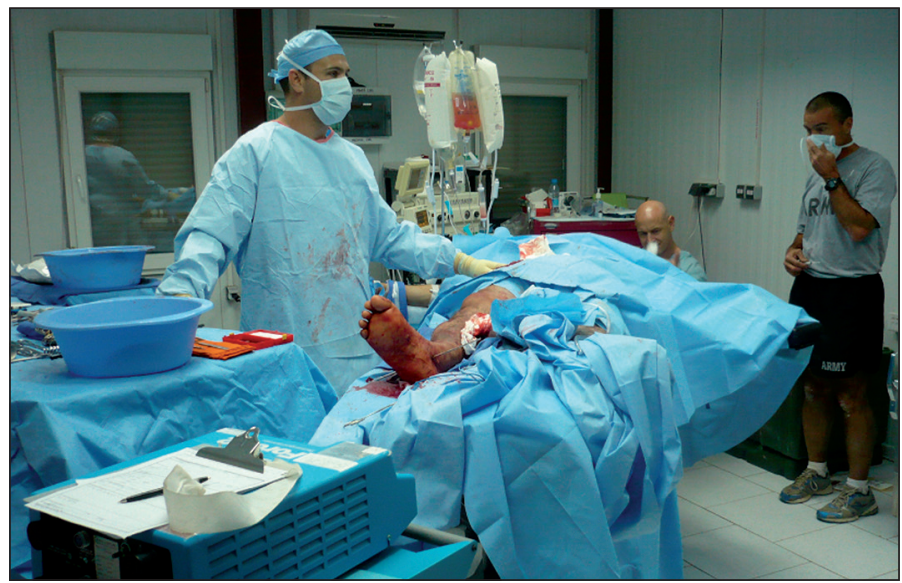

Figura 9. 2. ${ }^{\circ}$ Escalón sanitario PSB «Ruy González de Clavijo» (Qala i Now). Fotografía del autor.
La FSB «Camp Arena» de Herat (Figura 12) dispone de un segundo escalón sanitario con zona de filiación, triaje, contenedor de radiología, quirófanos, unidad de cuidados intensivos, salas de hospitalización, gabinete odontológico, psicológico,

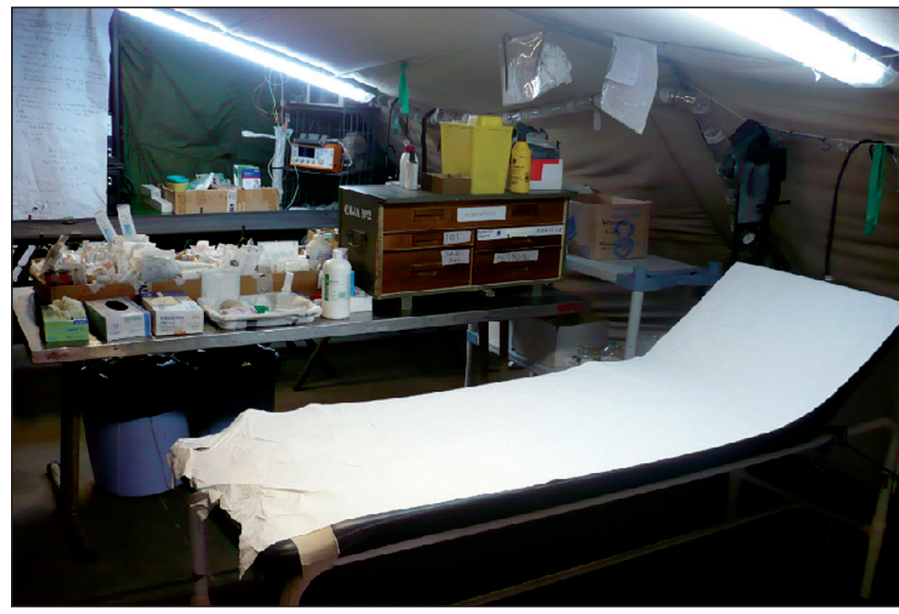

Figura 10. 1. ${ }^{e r}$ Escalón sanitario de la COP «Ricketts» (Muqur). Fotografía del autor.

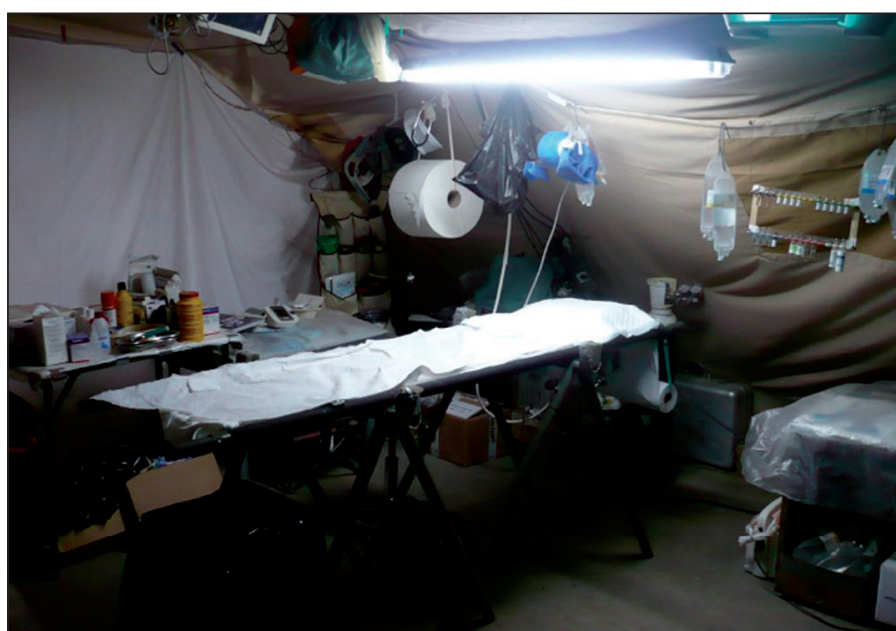

Figura 11. 1. ${ }^{e r}$ Escalón sanitario COP «Bernardo de Gálvez II» (Ludina). Fotografía del autor.

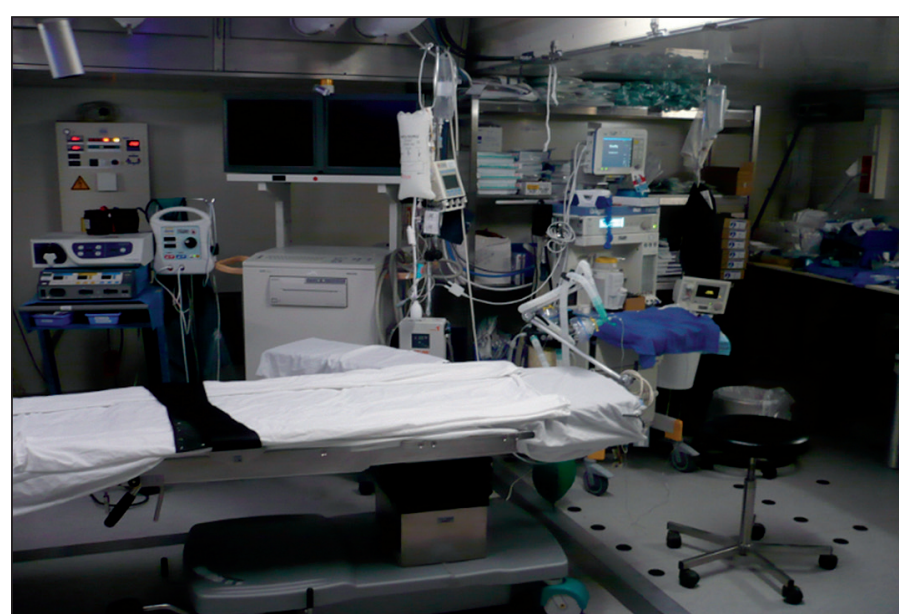

Figura 12. 2. ${ }^{\circ}$ Escalón sanitario FSB «Camp Arena»(Herat). Fotografía del autor. 
Despliegue y capacidades sanitarias en la región oeste de Afganistán (provincia de Badghis y Herat)...

Tabla 2. Equipamiento sanitario principal de la COP «Ricketts» (Muqur), COP «Bernardo de Gálvez II» (Ludina), PSB «Ruy González de Clavijo» (Qala i Now), FSB «Camp Arena» (Herat).

\begin{tabular}{|c|c|c|c|c|c|c|}
\hline Dispositivo & $\begin{array}{l}1 \text { Esc (Muqur) } \\
\text { España }\end{array}$ & $\begin{array}{c}1 \text { Esc (Ludina) } \\
\text { España }\end{array}$ & $\begin{array}{c}1 \text { Esc (QiN) } \\
\text { España }\end{array}$ & $\begin{array}{c}\text { Esc (QiN) } \\
\text { EE.UU }\end{array}$ & $\begin{array}{l}1 \text { Esc (Herat) } \\
\text { Italia }\end{array}$ & $\begin{array}{l}2 \text { Esc (Herat) } \\
\text { España }\end{array}$ \\
\hline Aspirador eléctrico & 1 & 1 & 2 & 2 & 1 & 6 \\
\hline Esterilizador quirúrgico & 2 & 2 & 2 & 2 & 1 & 4 \\
\hline Glucómetro & 3 & 3 & 3 & 4 & 2 & 3 \\
\hline Monitor de constantes & 1 & 1 & 2 & 5 & 2 & 3 \\
\hline Monitor desfibrilador & 1 & 1 & 2 & 2 & 4 & 4 \\
\hline ECG & - & - & 1 & 1 & 1 & 4 \\
\hline Ventilador & 2 & 1 & 2 & 4 & 1 & 6 \\
\hline Ventilador portátil & 1 & 1 & 1 & 2 & 1 & 5 \\
\hline Refrigerador/calentador & 1 & 1 & 1 & 1 & 1 & 2 \\
\hline Capnógrafo & - & 1 & - & 1 & 0 & 1 \\
\hline Ecógrafo & 1 & 1 & 1 & 1 & 1 & 1 \\
\hline $\mathrm{Rx}$ & - & - & 1 & 1 & - & 2 \\
\hline TAC & - & - & - & - & - & 1 \\
\hline Intensificador imagen & - & - & - & 1 & - & 1 \\
\hline Cama de triaje & 2 & 1 & 2 & 2 & 1 & 4 \\
\hline Cama hospitalización & - & - & 4 & 4 & 2 & 14 \\
\hline Cama de UCI & - & - & - & 2 & - & $4(+1)$ \\
\hline Quirófano & - & - & - & 1 & - & 2 \\
\hline Telemedicina & SÍ & Sí & SÍ & Sí & NO & SÍ \\
\hline
\end{tabular}

Esc: Escalón sanitario. QiN: Qal i Now.

despacho de veterinaria, almacenes de farmacia y veterinaria, planta generadora de oxígeno, contenedor con $\mathrm{TAC}^{13}$ y zona para el equipo de aeroevacuación entre otras. Para el contingente español esta instalación sanitaria además de tener funciones

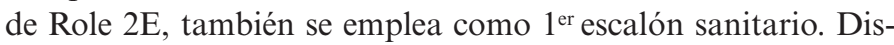
pone de aeropuerto con capacidad de aterrizaje de aeronaves que permitan la aeroevacuación estratégica y táctica. La distancia es de aproximadamente $1 \mathrm{~km}$ y para la evacuación de bajas se cuenta con ambulancias españolas de distinto tipo (2 Mercedes sprinter, 1 URO-VAMTAC blindada ${ }^{14}$, Iveco daily no blindada). Italia dispone de 2 lince ambulancia parcialmente blindados y 1 ambulancia Iveco no blindada).

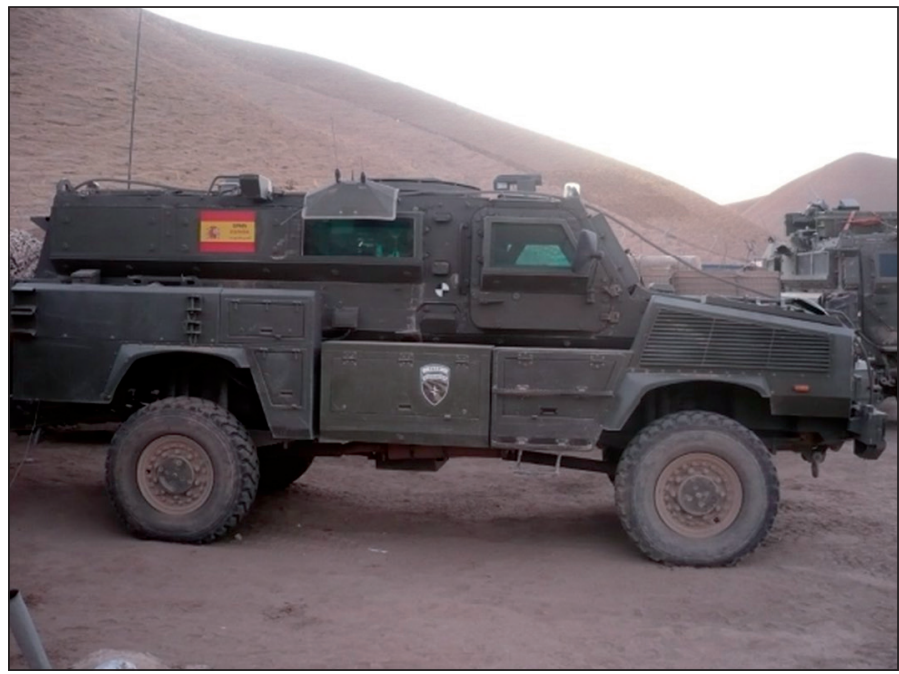

Figura 13. RG-31 ambulancia, vehículo blindado de las células de estabilización. Fotografía del autor.

\section{Material (Tabla 2)}

Los principales materiales y dispositivos sanitarios de cada una de las instalaciones se resumen en la tabla 2. Es necesario recalcar la posibilidad de establecer enlace de comunicaciones entre todas las instalaciones sanitarias gracias a la telemedicina ${ }^{15}$.

$\mathrm{El}$ abastecimiento de los escalones sanitarios de la provincia de Badghis y de Herat sigue canales logísticos diferentes debido a que los primeros dependen orgánica y funcionalmente del ejército de tierra ${ }^{16}$, mientras que el segundo depende del ejército del aire ${ }^{17}$. Hay vuelos regulares desde territorio nacional aproximadamente cada 15 días que permiten conseguir los niveles de recursos clase VIII adecuados.

\section{Función encomendada a cada escalón sanitario}

La función de cada uno de los escalones sanitarios y su capacidad es la correspondiente a la normativa OTAN ${ }^{18-20}$.

\section{Medios de evacuación}

\section{Terrestres: Célula de estabilización}

Con anterioridad a la realización de este artículo, los equipos de estabilización del ejército de tierra desplegados en Afganistán han empleado varios tipos de ambulancia: Iveco $40-10^{21}$, BMR ${ }^{22}$, URO-VAMTAC, lince, $\mathrm{TOM}^{23}$, pero en la actualidad utilizan RG3124(Figura 13) (Tabla 3). Este vehículo se desplegó en zona desde 2010 y está especialmente diseñado para soportar los efec- 
Tabla 3. Material sanitario del RG 31 ambulancia.

\begin{tabular}{|lc|}
\hline Descripción & Cantidad \\
\hline Botellas de oxígeno 10 L & 2 \\
Desfibrilador bifásico PHILIPS & 1 \\
Respirador automático OXILOG 2000 PLUS & 1 \\
Monitor constantes vitales PROPAQ & 1 \\
Aspirador secreciones & 1 \\
Tablero espinal & 1 \\
Inmovilizador cervical tipo dama de Elche & 1 \\
Cartera de camillero & 2 \\
Ampulario & 1 \\
Equipo de exploración Oftalmología/Otorrino & 1 \\
Camilla OTAN & 4 \\
Pulsioxímetro portátil & 1 \\
Capnógrafo & 1 \\
Chaleco inmovilizador kendrik & 1 \\
Juego de férulas neumáticas & 2 \\
Mochila SVA (circulatorio respiratorio) & 1 \\
Maletín de medicación general & 1 \\
\hline
\end{tabular}

tos provocados por los artefactos explosivos improvisados ${ }^{25}$. El principal material sanitario está especificado en la tabla, pudiendo transportar una baja en camilla y dos sentadas. El tiempo de evacuación terrestre entre Muqur y Qal i Now es de aproximadamente 150 minutos, entre Ludina y Qal i Now es sobre 180 minutos y de cerca de 10 horas entre Qal i Now y Herat.

\section{Aéreos: Equipo de aeroevacuación médico}

Los medios aéreos que dispone España en la región oeste de Afganistán son: un T10 «Hércules», dos helicópteros AS 232 «Superpuma» (Figura 14 y 15) (Tabla 4) y 3 helicópteros $\mathrm{CH}$ 47 «Chinook» ${ }^{1}$. Los que están configurados permanentemente en modalidad sanitaria son $\operatorname{los}$ «Superpuma ${ }^{26}$, aunque el resto de aeronaves podrían realizar funciones de evacuación si fuese necesario ${ }^{27-29}$

La capacidad de transporte del «Superpuma» es de 2 bajas en camilla y 5 ambulatorias, mientras que la del «Chinook» es de 24 camillas. Por su parte el avión T-10 «Hércules» cuenta con una capacidad máxima de evacuación de 92 bajas sentadas o 74 camillas.

Desde el año 2011 la FSB de Herat cuenta en su aeropuerto con una pista que permite el aterrizaje de aviones comerciales procedentes de $\mathrm{TN}^{30}$. Así mismo, la PSB de Qal i Now también ha mejorado la pista de su aeródromo consiguiendo una mayor seguridad en vuelo ${ }^{31}$. Estos avances permiten optimizar la evacuación táctica (entre dos escalones sanitarios de zona de operaciones) y estratégica (entre un escalón sanitario desplegado en zona de operaciones hasta el cuarto escalón ubicado en territorio nacional) ${ }^{32}$.

Por su parte, las fuerzas estadounidenses cuentan en estas dos provincias con 12 helicópteros UH-60 «Blackhawk», 5 en la PSB de Shindan, 3 desplegados en la PSB Camp Victory y 4 en la PSB de Qal i Now. Preferentemente realizan evacuaciones

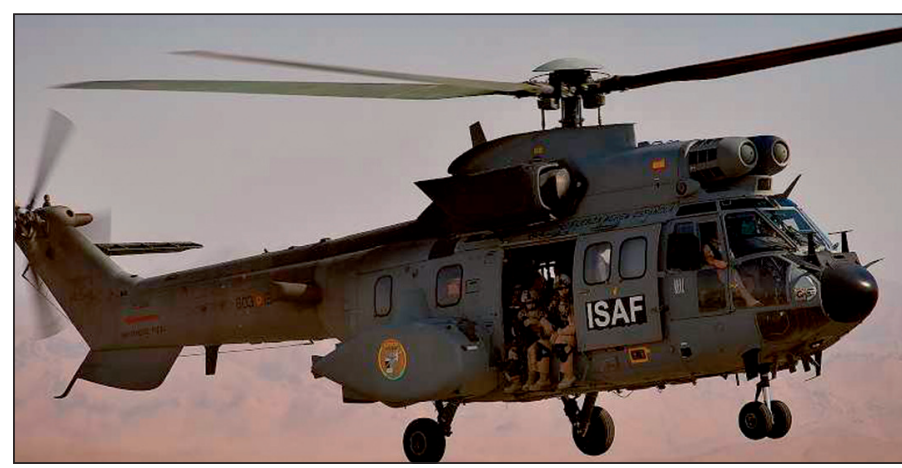

Figura 14. Helicóptero Superpuma de aeroevacuación médica español desplegados en la FSB «Camp Arena» (Herat).

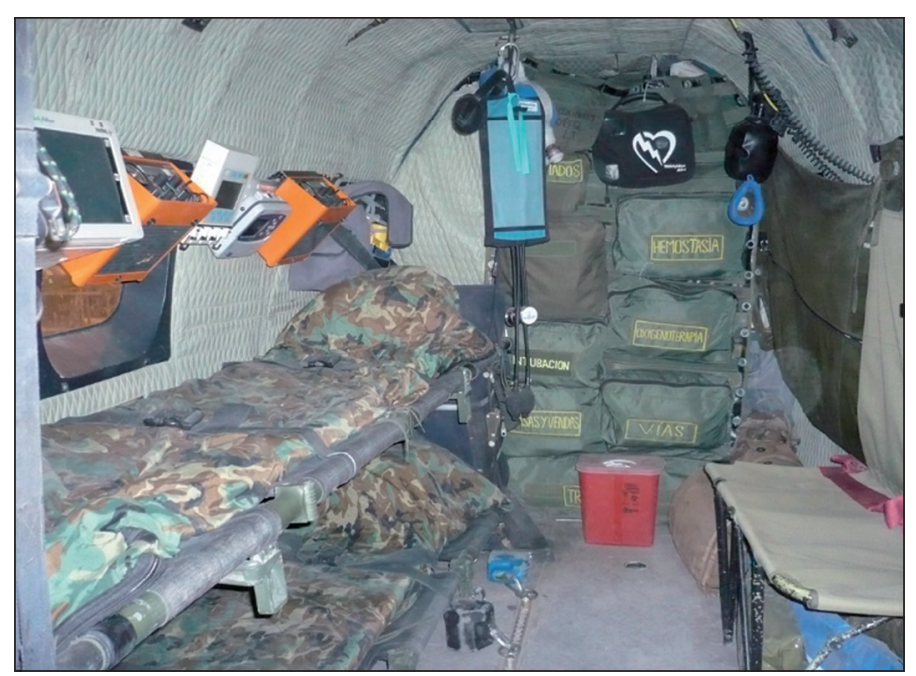

Figura 15. Interior del helicóptero AS 232 Superpuma de aeroevacuación médica. Fotografía del autor.

de tipo avanzado (entre el lugar del incidente y un escalón sanitario) y táctico. En cada uno de sus aparatos la tripulación con cometidos sanitarios es de 1 paramédico. Su capacidad de evacuación es de 3 bajas en camilla o 4 sentadas.

Italia dispone de un equipo de aeroevacuación médica con base en la FSB de Herat. Emplea un NH-90 como aeronave sanitaria. La tripulación está compuesta por un oficial médico y un suboficial enfermero. La capacidad máxima de evacuación en este aparato es de 12 bajas en camilla.

Si se decide solicitar una aeroevacuación sanitaria, desde el lugar del incidente hay que rellenar un documento (9 líneas) ${ }^{33} \mathrm{y}$

Tabla 4. Principal material sanitario del helicóptero AS 232 Superpuma de aeroevacuación médica español.

\begin{tabular}{|lc|}
\hline Descripción & Cantidad \\
\hline Monitor de constantes & 2 \\
Ventilador & 2 \\
Bomba de perfusión & 1 \\
Aspirador de secreciones & 1 \\
Manguito de presión de fluidoterapia & 1 \\
Desfibrilador & 1 \\
Capnógrafo & 1 \\
Camilla OTAN & 2 \\
\hline
\end{tabular}


una ficha de información médica (MIST) (Figura 16). Por experiencia de los autores, el tiempo de evacuación desde las aeronaves desplegadas en Qal i Now hasta el lugar del incidente dentro de la provincia de Badghis no es superior a 30 minutos, en caso de que las condiciones meteorológicas sean óptimas, la situación táctica no esté comprometida y la gravedad de la baja sea alta. El tiempo de respuesta de los equipos de aeroevacuación médica españoles es de $30 \mathrm{~min}$ (vuelo diurno) y $60 \mathrm{~min}$ (vuelo nocturno). Los equipos estadounidenses tienen un tiempo de respuesta de

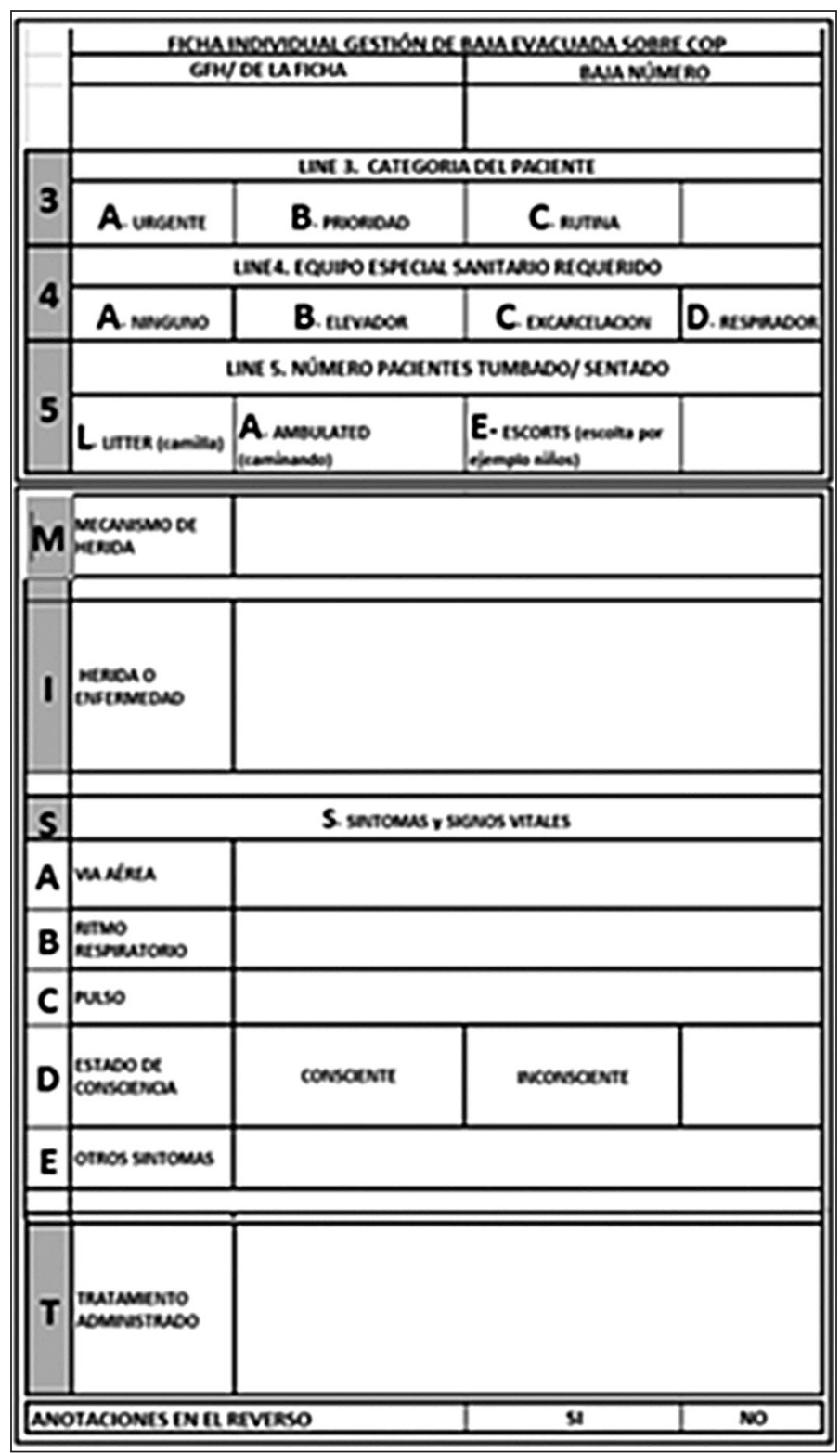

Figura 16. Parte del mensaje 9 líneas + MIST que cumplimenta un oficial del CMS. El resto de lineas son: 1. Localización (MGRS). 2. Indicativo \& Frecuencia: 6. Seguridad en zona de toma ( $N$-sin enemigo. E- enemigo en la zona. P-posible enemigo en la zona. X-zona peligrosa). 7. Método de señalización en zona de toma (A-paineles. B- bengala. C-humo. D-ninguno. E-otro). 8. Número de pacientes por nacionalidad / Categoría [A-(militares ISAF) $B$-(civiles con ISAF) C-(militares no ISAF) D-(Civiles no ISAF) E-(Enemigo/prisionero de guerra) F-(Niño) ] 9. Terreno / Obstáculos en zona de toma.
15 min (vuelo diurno) y 30 min (vuelo nocturno). El tiempo de evacuación entre Qal i Now y Herat es de 25 min en avión T-10 «Hércules» y aproximadamente 45 min en helicóptero. La evacuación mediante aeronaves de ala fija desde Herat a terceros escalones sanitarios en zona de operaciones (Mazar e Shariff o Camp Bastion) es de menos de 75 min. El vuelo de Herat a Madrid (4. ${ }^{\circ}$ escalón sanitario) es de 8 horas en avión Boeing del ejército del aire.

Se han establecido tres categorías $(\mathrm{A}, \mathrm{B}, \mathrm{C})$ de bajas, siendo Alfa la más crítica (tiempo de evacuación hasta 2. $\% 3$. $^{\text {er }}$ escalón de 1 hora), Bravo (tiempo de evacuación hasta $2 . \% / 3$. ${ }^{\text {er }}$ escalón 4 horas) y Charlie la más leve (tiempo de evacuación hasta 2.\% $/ 3$.r escalón 24 horas $)^{34}$.

\section{ACTIVIDADES REALIZADAS}

\section{Asistencial}

Durante el periodo de este artículo, se realizó la función de recuperación del personal (recogida de las bajas sanitarias y aplicación de los primeros cuidados médicos, clasificación de dichas bajas por gravedad, realización de la puesta en estado de evacuación, realizar las evacuaciones y tratamiento de las bajas sanitarias) y prevención sanitaria (mediante la adopción de medidas de medicina preventiva y educación sanitaria ${ }^{19}$ (Figura 17 y 18) (Tabla 5). Se destaca la atención de bajas de combate atendidos por oficiales del CMS en los diferentes escalones sanitarios desplegados en la provincia de Badghis durante el tiempo de misión (Tabla 6). Asimismo se realizaron los distintos reconocimientos médicos de fin de misión ${ }^{35}$. Además se colaboró en el planeamiento sanitario de las operaciones de la fuerza.

Durante el tiempo del estudio en el 2. ${ }^{\circ}$ escalón sanitario de Herat se realizaron 18 intervenciones quirúrgicas, fueron hospitalizados 46 enfermos y en la unidad de cuidados intensivos ingresaron 32 pacientes. Se realizaron 3 consultas mediante telemedicina.

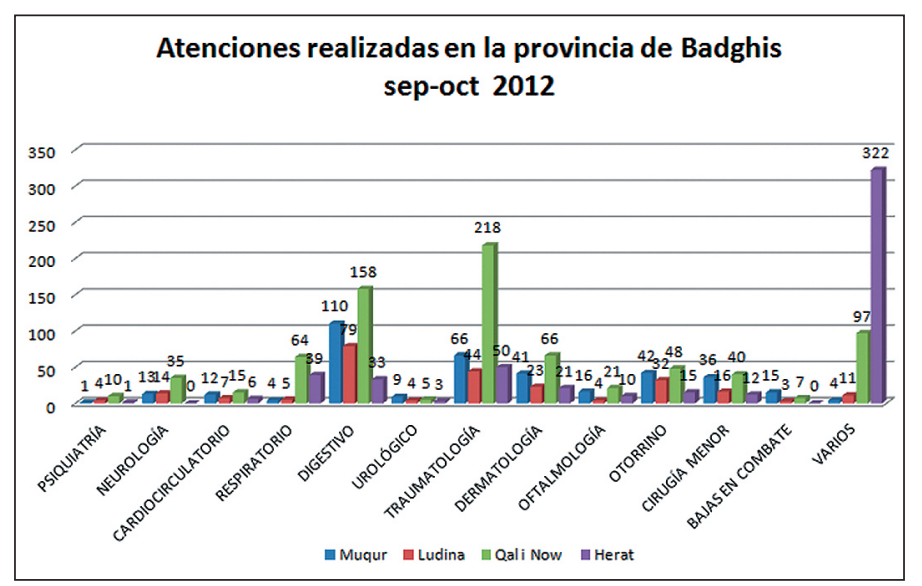

Figura 17. Número de consultas realizadas en el primer escalón sanitario de la COP «Ricketts» de Muqur y COP «Bernardo de Gálvez» de Ludina entre septiembre y octubre de 2012. Fuerza en lista de revista: Qal i Now (aprox. 600 efectivos), Herat (aprox. 300 efectivos), Muqur (aprox. 240 efectivos), Ludina (aprox. 240 efectivos). 


\section{Porcentaje de consultas realizadas en cada destacamento septiembre-octubre 2012}

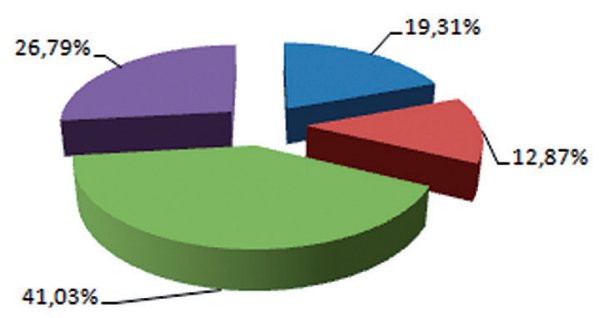

Muqur

- Ludina

Q Qal i Now

Q Herat

Figura 18. Porcentaje de las consultas totales que se han atendido en cada destacamento español entre septiembre y octubre de 2012. Fuerza en lista de revista: Qal i Now (aprox. 600 efectivos), Herat (aprox. 300 efectivos), Muqur (aprox. 240 efectivos), Ludina (aprox. 240 efectivos).

Concretamente el personal sanitario que integra la célula de estabilización se ha ocupado de la atención sanitaria que han precisado las fuerzas desplegadas fuera de la PSB, tanto en patrullas, convoyes u otro tipo de misiones. El personal de la célula de estabilización se ha encontrado en primera línea de contacto de la fuerza, atendiendo las contingencias sanitarias y las urgencias que han aparecido en el transcurso de las operaciones.

Las misiones realizadas por las células de estabilización son:

- Fuerza de rápida reacción (QRF). La célula de estabilización presta el apoyo sanitario a un contingente de guardia de 24 horas, de entidad sección dispuesto a cumplir una misión no prevista. El tiempo de reacción en la PSB es de 15 minutos, mientras que en las COP es el mínimo que permita embarcar en el vehículo y enlazar comunicaciones.

- Convoyes. La existencia de tres destacamentos españoles genera la necesidad de prestar un apoyo logístico entre ellos. Esto se consigue gracias a convoyes. Los oficiales de la célula colaboran en el planeamiento sanitario de forma previa.

- Patrullas (de seguridad, de presencia y de cooperación cívico militar-CIMIC-).

- Proyecto de asistencia médica a población civil (MEDCAP): Es una actividad con fines humanitarios sobre la población civil. Normalmente se envía una célula de estabilización fuera del destacamento para realizar esta tarea. Se atiende a población civil enferma y se reparte medicación no militar destinada a ayuda humanitaria.

Durante el periodo de estudio del artículo, el equipo de aeroevacuación español a bordo de los helicópteros Superpuma ha

Tabla 5. Número de consultas realizadas en los diferentes escalones sanitarios españoles de la provincia de Badghis y Herat de septiembre a octubre de 2012.

\begin{tabular}{|lcccc|}
\hline & $\begin{array}{c}\mathbf{1 .}^{\text {er }} \text { Esc. } \\
\text { Qal i Now }\end{array}$ & $\begin{array}{c}\mathbf{1 .}^{\text {er }} \text { Esc. } \\
\text { Muqur }\end{array}$ & $\begin{array}{c}\mathbf{1}^{\text {er }} \text { Esc. } \\
\text { Ludina }\end{array}$ & $\begin{array}{c}\mathbf{2 .}^{\mathbf{}} \text { Esc. } \\
\text { Herat }\end{array}$ \\
\hline Militares ISAF & 608 & 248 & 171 & 280 \\
Militares ANA & 10 & 41 & 32 & 21 \\
Civiles & 166 & 80 & 43 & 211 \\
Total & 784 & 369 & 246 & 512 \\
\hline
\end{tabular}

Datos obtenidos de documentación OTAN y libros de reconocimiento.
Tabla 6. Análisis de las 25 bajas en combate (por IED o Arma de fuego) atendidas por oficiales del CMS entre agosto y noviembre de 2012.

\begin{tabular}{|llcc|}
\hline & & n & $\%$ \\
\hline Sexo & Varón & 24 & 96 \\
& Mujer & 1 & 4 \\
\hline Edad & $<20$ años & 3 & 12 \\
& $20-40$ años & 22 & 88 \\
\hline Pertenencia & Civil & 3 & 12 \\
& ANA & 21 & 84 \\
& ISAF & 1 & 4 \\
\hline Agente lesional & IED & 21 & 84 \\
& Arma de fuego & 4 & 16 \\
\hline Zona lesionada $\left.*^{*}\right)$ & Cabeza & 10 & 40 \\
& Cuello & 4 & 16 \\
& Tórax & 3 & 12 \\
& Abdomen & 7 & 28 \\
& Miembros superiores & 13 & 52 \\
& Miembros inferiores & 19 & 76 \\
\hline Cirugía mayor & Sí & 14 & 56 \\
& No & 9 & 44 \\
\hline Evacuación $2 \mathrm{a}\left({ }^{* *}\right)$ & Sí & 15 & 60 \\
& No & 10 & 40 \\
\hline Fallecimiento & Sí & 7 & 28 \\
& No & 18 & 72 \\
\hline
\end{tabular}

(*) Hay 10 bajas $(40 \%)$ con lesión en un área anatómica, $6(24 \%)$ con lesión en dos áreas anatómicas, $3(12 \%)$ con lesión en tres áreas anatómicas, $5(20 \%)$ con lesión en cuatro áreas anatómicas y 1 baja (4\%) con lesión en cinco áreas anatómicas diferentes. $(* *)$ Evacuación 2. ${ }^{\mathrm{a}}$ a otra instalación sanitaria con mayor capacidad.

realizado 2 evacuaciones avanzadas, 8 tácticas, estabilizando a 8 bajas. Asimismo se han realizado dos aeroevacuaciones estratégicas con un total de cuatro bajas españolas hasta el Hospital Central de la Defensa «Gómez Ulla» (Madrid) ${ }^{36,37}$. Únicamente se ha trasladado 1 baja por tierra desde una COP hasta la PSB.

\section{Farmacia}

Se encargó de la obtención, almacenamiento, abastecimiento y mantenimiento de los recursos sanitarios necesarios. Se realizaron análisis de aguas y asesoramiento en las cuestiones relacionadas con la depuración, captación, suministro y transporte de la misma. Se colaboró con el servicio veterinario en el saneamiento ambiental y de las instalaciones ${ }^{19}$. En la PSB de Qal i Now: se recibieron 5 envíos logísticos, mientras que en Herat fueron 6. Durante el mes de octubre miembros pertenecientes a la Unidad de Apoyo Logístico Sanitario realizaron una revista al material e instalaciones sanitarias reparando los desperfectos y comprobaron los niveles de fármacos y dispositivos sanitarios.

\section{Veterinaria}

Se elaboraron planes y medidas de higiene y seguridad alimentaria. Se revisaron los planes de gestión y control de la higie- 


\section{Despliegue y capacidades sanitarias en la región oeste de Afganistán (provincia de Badghis y Herat)...}

ne y saneamiento ambiental de las bases. Se prestó apoyo sanitario de los animales de interés militar de las bases, así como de la prevención de enfermedades zoonósicas y riesgo de animales no controlados sanitariamente ${ }^{19}$. Como dato reseñable en la PSB de Qal i Now se realizó apoyo civil (desratización del mercado local, vacunación animal, mentorización a veterinarios locales, información por radio local de medidas básicas de prevención sanitaria...). En la FSB de Herat se realizaron las mismas actividades de higiene y seguridad alimentaria, así como a animales de interés militar y medidas de desinfección de aeronaves.

\section{Psicología}

Se realizaron tareas preventivas de la aparición de estrés asociado a la operación o de cualquier conducta disruptiva durante la misión ${ }^{19,38}$. En Qal i Now se realizó en 32 efectivos atención psicológica (distimia $27 \%$, problemas familiares $19 \%$, ansiedad $15 \%$, trastorno ansioso-depresivo $9 \%$, trastorno de personalidad $6 \%$, trastorno adaptativo $3 \%$, trastorno del sueño $3 \%$, trastorno por estrés postraumático 3\%, trastorno psicosomático 3\%). Asimismo se realizó la evaluación y optimización del estado de moral del personal interviniente en la misión, un estudio de satisfacción del personal militar con los servicios de la base y se realizaron talleres de relajación. Por ejemplo, en la PSB de Qal i Now se valoró mediante un cuestionario psicológico a todos los efectivos, se informó al jefe de la fuerza de los resultados obtenidos y se colaboró en la realización de avisos preventivos para mantener la atención hasta el final de la misión. El apoyo psicológico en Herat fue de 57 asistencias (ansiedad 35\%, depresión, problemas profesionales $35 \%$, estrés agudo $10 \%$, trastorno adaptativo $10 \%$, otros $10 \%$ ).

\section{Odontología}

El apoyo odontológico se realizó fundamentalmente en el 2. ${ }^{\circ}$ escalón sanitario de Herat. El número de consultas odontológicas realizadas fue de 322 consultas (obturación 40\%, endodoncias 30\%, extracción dental 20\%, fijación de coronas 10\%). Desde la PSB de Qal i Now se enviaron a 35 efectivos al servicio odontológico de la FSB de Herat. Desde el año 2012 se dispone de ortopantomografo en el gabinete odontológico.

\section{LECCIONES IDENTIFICADAS}

La atención sanitaria prestada en las bases españolas ha sido menor que en anteriores despliegues. En un artículo ${ }^{39}$ se describe un total de 911 y 1.832 asistencias en Qal i Now correspondientes a las agrupaciones ASPFOR XX y XXIV respectivamente. Durante el tiempo analizado en este estudio, la cifra de asistencias en esta misma base fue de 784. Aunque la duración y la población de los anteriores contingentes es algo superior a la nuestra, la disminución en el número de asistencias puede ser debido a un incremento de la actividad realizada por los médicos militares y civiles afganos desplegados cerca de nuestros destacamentos. La gastroenteritis aguda vírica ha sido la infección más prevalente en nuestro estudio. Aunque ha habido antece- dentes de brotes epidemiológicos entre las fuerzas desplegadas en Afganistán ${ }^{40,41}$, este cuadro digestivo ha sido limitado, leve y ha remitido en la mayoría de los casos con medidas higiénicas y sintomáticas. El número de consultas (una única consulta) por medio de telemedicina desde los primeros escalones también ha disminuido respecto a contingentes anteriores ${ }^{42}$. La presencia durante nuestro despliegue de un oficial médico diplomado en medicina interna, uno en traumatología, dos en anestesiología y reanimación, uno en psiquiatría, uno en medicina de familia y dos en medicina general puede que haya provocado el menor uso de este dispositivo. Es relevante el elevado número de bajas en combate (25) atendidas por oficiales del CMS español en los distintos destacamentos durante el tiempo del estudio. Este dato puede ser la consecuencia de las operaciones ofensivas realizadas por el contingente español y apoyadas por el ejército afgano desde septiembre a noviembre de $2012^{43-45}$. El perfil de la baja más prevalente que se nos ha presentado es similar al de experiencias previas ${ }^{46}$ : varón, entre 20 y 30 años, miembro del ejército nacional afgano. El agente lesional predominante sigue siendo el IED ${ }^{47}$ y al igual que en otros estudios la zona anatómica más afectada son los miembros inferiores ${ }^{48}$. Debido a la necesidad de estabilizar y tratar este tipo bajas, consideramos necesario normalizar los procedimientos, materiales y fármacos emplea$\operatorname{dos}^{49} \mathrm{e}$ implementar la formación médica en los cursos sanitarios previos al despliegue en zona de operaciones ${ }^{50}$, priorizando los conocimientos de sedoanalgesia en el politraumatizado militar, la actualización en cirugía ${ }^{51}$ y resucitación del control del daño ${ }^{52}$.

Principalmente la evacuación se realiza por medios aéreos. Este hecho se debe fundamentalmente al tipo de orografía de la zona de operaciones, a la pobre red de comunicaciones terrestres existente, a la superioridad aérea de las fuerzas de ISAF, al componente táctico de esta operación, a la disponibilidad de medios aéreos especializados en evacuación de bajas y a la necesidad de cumplir un plazo operatorio impuesto por la OTAN ${ }^{32}$. El primer artículo que analiza el número de evacuaciones realizadas por el equipo de aeroevacuación español desplegado en Herat muestra que entre febrero y julio de 2007 se evacuaron 43 bajas en combate (arma de fuego o IED) en 31 vuelos $^{42}$. En un estudio posterior $^{26}$, se concreta que el número de este tipo de bajas evacuadas por el equipo español en 2010 ascendió a 151. Durante el periodo de nuestro informe, el número de bajas en combate evacuadas por la tripulación aérea española ha sido de 8 , notablemente inferior a otros periodos. Este hecho posiblemente se deba al nuevo despliegue en nuestra zona de responsabilidad de los equipos de aeroevacuación estadounidenses.

Las evacuaciones terrestres hasta un escalón sanitario han sido anecdóticas (1 baja). La preferencia por emplear los medios aéreos, las características de la zona de operaciones y las peculiaridades del vehículo blindado ambulancia RG-31 han provocado que las bajas evacuadas por este medio sean escasas. Hemos comprobado como con el nuevo RG-31 se ha mejorado ostensiblemente en seguridad para la tripulación y la baja ${ }^{25,53}$, sin embargo es complicado emplearlo como medio de evacuación. Siempre que lo ha permitido la situación táctica, los autores hemos preferido que la atención del herido se efectuase a pie de vehículo, desembarcando fuera de la ambulancia el material y dispositivos que fuesen necesarios, poniendo en estado de evacuación a la baja sobre el terreno, para finalmente transportarla a mano hasta 
la zona de toma de helicópteros más cercana. Esta secuencia se ve facilitada gracias al empleo de la nueva cartera del camillero. Los autores consideramos necesario la normalización de una bolsa de dimensiones ligeramente superiores a las de la actual cartera de camillero que no suponga un incremento excesivo en el peso a transportar y que permita su empleo por el personal facultativo.

Otro punto de interés es la necesidad de que los oficiales médicos asesoren al mando en el planeamiento de las operaciones ${ }^{19}$. Esta función es fundamental en los actuales despliegues y escenarios tácticos asimétricos ${ }^{54}$ y llega a ser reconocida por los propios oficiales del Cuerpo General de las Armas ${ }^{55}$.

La forma física de los oficiales del CMS debe ser adecuada ${ }^{56}$, ya que durante las misiones realizadas fuera de los destacamentos se les obliga a transportar armamento, casco, chaleco de protección balística y equipo individual con un peso aproximado de $20 \mathrm{Kg}^{46,57}$. A esto se suma la necesidad de contar con un nivel óptimo de idioma inglés ya que se trabaja en un ambiente multinacional y esta es la lengua oficial en el seno de la OTAN.

Los autores hemos comprobado la importancia que el sanitario ejerce dentro del equipo médico. Esta observación ya ha sido descrita con anterioridad ${ }^{58-60}$. Creemos que la figura del sanitario debe promoverse técnica y numéricamente al igual que en otros ejércitos de nuestro entorno ${ }^{61}$. En este sentido consideramos meritorio la formación teórico-práctica que se le está impartiendo en las distintas unidades ${ }^{62}$.

El abastecimiento de recursos sanitarios es una parte crítica del apoyo sanitario en las operaciones ${ }^{16,63}$. Dada la distancia desde territorio nacional, el transporte de medicamentos y productos sanitarios se realiza por vía aérea ${ }^{64}$. La frecuencia de los vuelos logísticos es similar a anteriores contingentes ${ }^{42}$. Sin embargo, el aumento en la atención de bajas en combate ha provocado la necesidad de incrementar el flujo logístico sanitario de determinados productos en la provincia de Badghis. La medicación más demandada fueron opiáceos, hipnóticos, benzodiacepinas, relajantes musculares y ketamina. Los dispositivos más solicitados pistolas intraóseas BIG y el nuevo botiquín individual del combatiente. Estas peticiones coinciden con la experiencia obtenida anteriormente $\mathrm{e}^{65-67}$.

Tal y como describe Zamanillo ${ }^{68}$, hemos comprobado sobre el terreno que el transporte de medicamentos resulta cualificado y que los elaborados fabricados en el Centro Militar de Farmacia de la Defensa no llegan alterados a la zona de operaciones a pesar de las temperaturas extremas que hemos padecido.

En esta zona de operaciones, es necesario un especial control de los vectores de transmisión de enfermedades infectocontagio$\operatorname{sas}^{69,70}$. Que los autores conozcan durante el periodo de estudio no se ha presentado ningún caso de paludismo, pero se ha seguido la labor propuesta en estudios entomológicos previos ${ }^{71}$ de mantener las medidas preventivas necesarias para evitar el contagio. Asimismo, se ha realizado un control de vectores en la base de despliegue que minimice la posibilidad de transmisión de enfermedades ${ }^{72}$. El impacto del control de plagas en la población civil y la mentalización realizada a veterinarios locales ha sido muy positivo.

Hemos comprobado un interés creciente de los efectivos desplegados por la nutrición. Según Lisbona ${ }^{73}$, el objetivo de la alimentación en zona de operaciones es proporcionar una suficiente energía, ingesta proteica, vitamínica, sales minerales y tolerancia intestinal para mantener al combatiente en las me- jores condiciones físicas y mentales. En un artículo ${ }^{74}$ publicado en la Revista Ejército se ha sugerido la necesidad de disponer de raciones de combate con un menú vegetariano. Estas mejoras en las raciones pueden ser importantes debido a que por la situación táctica planteada durante este despliegue, muchos de los efectivos nos hemos visto en la necesidad de recurrir a estas raciones durante largos periodos de tiempo.

El aspecto psicológico del militar desplegado en zona de operaciones en donde se incrementa la posibilidad de vivir una situación de emergencia puede ser primordial a la hora de culminar una misión ${ }^{75}$. El número de casos atendidos en el servicio de psicología de Qal i Now ha sido de 32, mientras que en el de Herat fue de 44. Estos resultados concuerdan con el artículo de Martínez $^{76}$ que revisa 172 pacientes en 4 años atendidos en la FSB de Herat. Un detalle que hemos comprobado es la disminución de manifestaciones externas de ansiedad provocadas por la misión. En ello se nota el grado de veteranía de la fuerza (el 65\% y 49,4\% de los mandos y tropa respectivamente del Batallón de Maniobra de la Bandera de Infantería Ligera Paracaidista III «Ortíz de Zárate» han sido desplegados con anterioridad en el exterior. El 79,5\% de la tropa ha cumplido un compromiso de más de 2 años con las Fuerzas Armadas y 65,5\% de la misma, más de 3 años de compromiso). La experiencia en este tipo de situaciones es un factor positivo que reduce el riesgo de fricciones y de conflictividad que hayan podido aparecer en anteriores misiones, a lo que habría que añadir el alto grado de mentalización y de disciplina del personal participante ${ }^{56}$.

Durante el periodo de estudio se realizó una encuesta psicológica al contingente español desplegado en Qal i Now. Los resultados mostraron un alto nivel de moral en la fuerza, una mejora en los hábitos de comportamiento y una disminución en el consumo de alcohol. Este dato ya se ha ido haciendo patente en las fuerzas armadas españolas desde los últimos años ${ }^{77}$.

En el actual despliegue sanitario en la zona de operaciones del oeste de Afganistán, únicamente hay un oficial odontólogo en el 2. ${ }^{\circ}$ escalón sanitario de Herat. Sin embargo es necesaria la atención odontológica ya desde el primer escalón. Esto lo contempla la doctrina OTAN ${ }^{78}$. En nuestro estudio, se realizaron 322 asistencias en Herat. Se aprovecharon vuelos logísticos entre los distintos destacamentos para que los pacientes pudieran ser valorados y tratados por el oficial odontólogo. Según el estudio de Mombiedro $^{79}$, políticas preventivas de diagnóstico precoz y de asistencia personalizada adaptadas al medio militar pueden ser clave para desplegar tropas que no ocasiones emergencias en futuras operaciones. Estos datos concuerdan con las valoraciones del oficial odontólogo desplegado durante el estudio. Este argumenta que se está produciendo una mejora sustancial en la salud bucal de los efectivos militares españoles desplegados en zona de operaciones, ya que la mayoría de las atenciones odontológicas realizadas en este colectivo se deben a problemas puntuales aparecidos durante el transcurso de la misión.

\section{CONCLUSIÓN}

Desde 2002 las fuerzas armadas españolas integradas en la operación ISAF están desempeñando una importante labor en Afganistán. A mediados de 2008 el centro de gravedad de las 


\section{Despliegue y capacidades sanitarias en la región oeste de Afganistán (provincia de Badghis y Herat)...}

operaciones pasó desde la provincia de Herat a la de Badghis. Este cambio ha provocado una modificación en el despliegue de la sanidad militar española en esta zona de operaciones. Las principales lecciones identificadas por los autores de agosto a noviembre de 2012 se resumen en la necesidad de implementar el papel del sanitario dentro del equipo médico, normalizar el material, procedimientos y fármacos empleados sobre las bajas en combate, destacar la seguridad proporcionada por los nuevos vehículos RG31 ambulancia y continuar eligiendo preferentemente la aeronave sanitaria como principal medio de evacuación hasta el correspondiente escalón sanitario. Asimismo consideramos prioritario que los oficiales del CMS mantengan una formación técnica actualizada, un estado físico aceptable y un adecuado nivel de lengua inglesa para poder desempeñar y cumplir de forma adecuada la misión encomendada.

\section{AGRADECIMIENTOS}

A los oficiales médicos, veterinarios, farmacéuticos, psicólogos, odontólogos y enfermeros, así como a los suboficiales y tropa encuadrada en las diferentes instalaciones sanitarias que con su ayuda desinteresada han permitido la realización de este estudio.

\section{BIBLIOGRAFÍA}

1. Domíngez J. Operaciones de las fuerzas armadas en el exterior. Revista española de defensa 2012; 282:18-21.

2. Fernández Arrojo JJ. Infraestructuras en operaciones, la PSB en Qala e Now. Revista ejército 2012; 851:85-91.

3. Gonzalo B. Afganistán: cuenta atrás. Tierra, boletín informativo del ejército español 2012; 197: 7-10.

4. Díaz P. La BRIPAC en Afganistán. Revista española de defensa 2012; 286:16 7.

5. Ferragut M. Medio año en Afganistán. Diario de Mallorca 2012, 14 octubre 2012:12-13.

6. Montánchez E. Ruta Lithium, misión cumplida. Revista española de defensa 2012; 287: 15-21.

7. Acuerdo técnico entre el contingente nacional español e italiano. http://www. ejercitodelaire.mde.es/ea/

8. NOP 103 de septiembre de 2011 que regula el funcionamiento del servicio de asistencia sanitaria de la AGT.

9. Mediavilla C, Martín G, Lorente B. Especialidades fundamentales del Cuerpo Militar de Sanidad en misiones internacionales. Revista aeronáutica y astronáutica 2010; 798: 974-79.

10. Moreno E. Gestión de personal en el CMS EA en relación a misiones. Revista aeronáutica y astronáutica 2010; 798: 970.

11. Directiva 17/11 de JEMAD punto 5.3.

12. Instrucción 7-10 de JEMAD

13. La dirección de sanidad hace entrega de un escáner al hospital de la base de apoyo avanzado de Herat. Noticias, 2009 [citado 20 Oct 2010]. Disponible en:www.ejercitodelaire.mde.es

14. Ambulancia blindada del Ejército del Aire destinada a servicios sanitarios en Herat. Noticias, 2009 [citado 20 Oct 2012]. Disponible en: www.ejercitodelaire.mde.es

15. Nota de prensa Ministerio de Defensa, militares españoles en Afganistán disponen de equipos de telemedicina. 27/11/2011.

16. García D. El apoyo logístico en las operaciones. Revista ejército 2012; 851: 54-63.

17. Lázaro J. Adquisición de recursos sanitarios en el Ejército del Aire. Revista aeronáutica y astronáutica 2010; 798: 968-9.

18. Campillo Laguna J.R. Bases Históricas del escalonamiento del Servicio de Sanidad en operaciones. Sanid. mil. 2008; 64(1): 43-51.
19. Ejército de Tierra español, mando de adiestramiento y doctrina. Sanidad en Operaciones PD4-616. Madrid: Dirección de Servicios Técnicos del Ejército; 2011.

20. Laguardia JC, Díez A, Rodrigo CF, Pérez CA, Fernández MD, Alcheikh MM. Definición de un ROLE 1 sobre contenedor «6 en 1» de la Unidad Médica Aérea de Apoyo al Despliegue (UMAAD) Zaragoza. Sanid Mil 2012: 68(1):40-3.

21. Navarro Suay R, Plaza Torres JF, Mota Ortega JM, Gutiérrez Manzanedo F, Jiménez Moreno R, Redondo Castán LC. Medios de evacuación en la Infantería de Marina española. Lecciones sanitarias aprendidas de Bosnia-Hercegovina, Irak, Haití y Líbano. Revista de Sanidad Militar 2008;64 (1): 31-40.

22. Relanzón López J. Ambulancias acorazadas B.M.R. 6x6. Revista de medicina militar 1987; 43 (5): 492-3.

23. Crego Vita DM, Vinué Jiménez C, Álvarez Suarez F. Transporte Oruga de Montaña ¿Vehículo de transporte sanitario? Revista de Sanidad Militar 2007; 63 (2):146-9.

24. RG 31 ambulancia, descripción y lecciones aprendidas del nuevo vehículo blindado sanitario en Afganistán. [En prensa]. Revista de Sanidad Militar. 2012.

25. Dos explosiones de IED's en Afganistán al paso de dos RG-31 españoles. [citado 5 may 2011]. Disponible en www.ateneadigital.es

26. Munayco Sánchez AJ, Navarro Suay R, de Nicolás Álvarez MA. Modelo español de MEDEVAC, experiencia en Afganistán. Sanid Mil 2012: 68(3):164-67.

27. Laguardia Chueca JC, Pérez Ribelles V. C-130 Hércules, empleo sanitario. Revista de medicina militar 1987; 43 (5): 492-3.

28. García J. El servicio de sanidad en las operaciones aeromóviles. Medicina Militar 1985; 41 (4): 441-3.

29. Hernando AE, Estella JF, Perales N, Coma R, Mateos J. Evacuación aérea sanitaria en el Ejercito. Med. Mil. 1985; 41 (2): 214-21.

30. Inaugurada la ampliación del aeropuerto de Herat. [citado 21 feb 2011]. Disponible en www.ejércitodelaire.mde.es

31. La pista de aterrizaje de Qala e Now alcanza los 5000 movimientos en menos de siete meses. [citado 10 ago 2010]. Disponible en www.ejércitodelaire.mde.es

32. Maimir F, Hernández A. Metodología y Material en el Transporte Sanitario militar en Zona de Operaciones. Medicina Militar 2006; 62 (1): 32-8.

33. Betegón A. Evacuación sanitaria en área/zona de operaciones. Med. Mil. 2006; 62 (3): 159-61.

34. SOP 307 de 14 feb 2011 .

35. Instrucción técnica número 6 de 15 de Julio de 2011 de la Inspección General de Sanidad de la Defensa, sobre requisitos sanitarios exigibles al personal militar que se desplace a zonas de operaciones. Anexo 3 cuestionario, Sanidad Militar, Ministerio de Defensa.

36. NOP 105 de septiembre de 2011 de asistencia y evacuación sanitaria.

37. Un militar español herido y cinco muertos de la insurgencia en varias horas de hostigamiento en la ruta Lithium [citado 29 ago 2012]. Disponible en www.ateneadigital.es

38. NOP 104 de septiembre de 2011 de asistencia psicológica

39. Amellugo J. Médicos militares en el Role 1 de Afganistán. Revista ejército 2012; 853: 92-9.

40. Maimir F, García JM, Sánchez MA, García RI, Gutiérrez C. Brote de gripe A H1N1 en la base española de Camp «Arena» (Herat, Afganistán) durante julio y agosto de 2009, parte I: características clínicas y reducción de la duración de la fiebre con el uso de oseltamivir. Sanid mil 2011; 67(1): 6-10.

41. Maimir F, Sánchez MA, García JM, García RI, Torner A. Brote de gripe A H1N1 en la base española de Camp «Arena» (Herat, Afganistán) durante julio y agosto de 2009, parte II: medidas clínicas y preventivas. Sanid mil 2011; 67(1): 31-5.

42. Navarro R, Bartolomé E, Jara I, Oreja A, González G. Capacidades y asistencia sanitaria realizada por el ROLE-2 español en la FSB de Herat (Afganistán) desde febrero a julio del 2007. Sanid. Mil. 2008; 64 (2): 98-104.

43. La «Operación Estaca» despeja la ruta Lithium. [citado 31 ago 2012]. Disponible en www.ateneadigital.es

44. Las fuerzas españolas y el ejército afgano completan la operación «ontur» para reducir la insurgencia en Muqur. [citado 14 sep 2012]. Disponible en www.ateneadigital.es

45. Las tropas españolas en Afganistán combaten con cien insurgentes en la ruta Lithium. [citado 8 oct 2012]. Disponible en www.ateneadigital.es

46. Navarro R. Bajas por arma de fuego y explosivos. Experiencia del Hospital Militar español desplegado en Herat (Afganistán) 2005-2008 [tesis doctoral]. Madrid: Universidad Autónoma de Madrid; 2009. 
47. Navarro Suay R, Hernández-Abadía de Barbará A, Gutiérrez Ortega C, Tamburri Bariain R, Bartolomé Cela E, Gilsanz Rodríguez F. Análisis del agente lesivo en la baja de combate. Experiencia de la Sanidad Militar española desplegada en Herat (Afganistán). Sanid mil 2011; 67(1):18-24.

48. Navarro Suay R, Hernández-Abadía de Barbará A, Gutiérrez Ortega C, Bartolomé Cela E, Tamburri Bariain R, Gilsanz Rodríguez F. Análisis de la topografía lesional en la baja de combate. Experiencia de la Sanidad Militar española desplegada en Herat (Afganistán). Sanid mil 2011; 67 (2):78-83.

49. Inspección General de Sanidad. Manual de asistencia al paciente crítico de las Fuerzas Armadas. Madrid: Imprenta ministerio de defensa; 2009.

50. Departamento de Medicina y Enfermería. Curso de Soporte Vital Avanzado en Combate. Escuela Militar de Sanidad. Madrid, 3-7 Octubre 2011.

51. De la Torre. Cirugía de control de daños/cirugía primaria. Sanid mil 2007; 63 (2): 141-5.

52. Jiménez JM, Pérez JM, Navarro R, Gómez M, Monsalve JA, Peyró R. Reanimación de control de daños en el paciente adulto con trauma grave. Rev. Esp. Anestesiol Reanim 2012; 59(1):31-42.

53. Presentación del nuevo modelo de ambulancias RG-31 (NYALA), por la unidad de apoyo logístico sanitario. Boletín informativo de Sanidad Militar. $2010 ; 4: 2$.

54. Alsina FJ. Consideraciones sobre el apoyo sanitario en combate urbano. Med Mil 2006; 62: 230-44.

55. Vega JA. Aquí Romeo siete: emergencia, se solicita apoyo sanitario urgente, cambio. Revista ejército 2010; 836: 72-6.

56. Fernández E. ROLE-1. Guardián de la Salud. La Legión 2009; (506): 24-9.

57. Knapik J, Reynolds K. Load carriage in military operations. Border institute monograph series 2012. Washington.

58. Guiote MJ. La sanidad operativa, una necesidad imperiosa. Revista ejército 2012; 851: 64-73.

59. Selva E. El nuevo hospital de campaña. Revista ejército 2012; 851: 74-83.

60. Selva E. Análisis de la situación de la Sanidad Militar, propuestas ante la crisis. Sanid mil 2009; 65 (4): 261-3.

61. Amellugo J. El futuro no tan incierto de la sanidad militar en misiones de paz. Sanid mil 2012; 68(2):129-30.

62. Curso de Sanitario en Operaciones (FSET-3). Ministerio de Defensa. Dirección general de relaciones institucionales. 2011.

63. Aguilar A, Astudillo JM, Juste F. El servicio de farmacia en la UMAD. . Revista aeronáutica y astronáutica 2000; 693: 374-7.

64. Bedate AF, Apoyo farmacéutico a las Fuerzas Armadas. Revista ejército 2011; 838: 62-9.

65. Navarro R, Castillejo S. Análisis de la analgesia en la baja de combate. Experiencia de la Sanidad Militar española. Sanid mil 2012; 68(1): 7-15.

66. Navarro Suay R, Bartolomé Cela E, Hernández-Abadía de Barbará A, Tamburri Bariain R, Rodríguez Moro C, Olivera García J. El acceso intraóseo para fluidoterapia en situación de combate. Experiencia de la sanidad militar española en Afganistán. Rev Esp Anestesiol Reanim. 2011; 58: 85-90.

67. Martín-Bilbatúa A. Tarborta E. Los diez minutos de platino. Revista Española de Defensa 2012 (7):44-5.

68. Zamanillo A, Verón M, Juberías A, Cabrera JL, Mañes JP. Condiciones de transporte de medicamentos en misiones internacionales de las Fuerzas Armadas, diseño de un estudio de estabilidad específico para operaciones. Sanid mil 2012; 68(3): 149-58.
69. Rodríguez-Marín JL. Misiones de apoyo sanitario (veterinaria) en la unidad médica de apoyo al despliegue aéreo. Revista aeronáutica y astronáutica 2000; 693: 378-9.

70. Moreno LA. Apoyo veterinario a las Fuerzas Armadas. Revista Ejército 2011; 838: 71-7.

71. Lacasa J, Melero R, Molina R, Presa MJ, Alcón A, Pérez A. Muestreo entomológico en zona de operaciones: ensayo piloto en la base de apoyo avanzado de Herat (Afganistán). Sanid mil 2010; 63(3):163-7.

72. Lacasa J. Introducción de mosquitos vectores de enfermedades por medio del tráfico aéreo, importancia de los sistemas de vigilancia entomológica y su aplicación en las Fuerzas Armadas. Sanid mil. 2009; 65(4):237-45.

73. Lisbona A, Rosado JA. Alimentación en zona de operaciones y riesgo cardiovascular. Sanid mil 2012; 68(3): 138-9.

74. Miguel FJ. Menú vegetariano para raciones individuales de combate. Revista Ejercito N.829. Mayo 2010.

75. Martínez JA. Aspectos psicológicos de la supervivencia en operaciones militares. Sanid mil 2011; 67(1): 43-8.

76. Martínez JA. Análisis descriptivo del personal militar español asistido por el Servicio de Psicología del Role 2 de Herat, Afganistán. Sanid mil 2012; 68(3):165-9.

77. Martínez M, Alonso V, Taranco M, Gutiérrez C. Patrón de consumo, actitudes y percepción del riesgo de alcohol de los militares profesionales de tropa y marinería de las Fuerzas Armadas españolas. Sanid mil 2009; 65(4): 221-30.

78. STANAG 2453 Tratamiento odontológico y maxilofacial en los Role 1-3.

79. Mombiedro R, Llena C. Caries en una población militar española. Sanid mil 2011; 67(1):36-42.

80. España envía un equipo de cirugía al hospital Role 2 de Herat (Afganistán). [citado 14 nov 2012]. Disponible en www.ateneadigital.es

\section{ACRÓNIMOS}

ANA: Ejército nacional afgano.

ASPFOR Fuerza española para Afganistán.

BMR: Blindado Medio sobre Ruedas.

CATO: Terminal aérea combinada.

CIMIC: Colaboración Cívico-militar.

CMS: Cuerpo Militar de Sanidad.

COP: Posición avanzada de combate.

FSB: Base de apoyo avanzado.

HELISAF: Destacamento de helicópteros del ejército del aire integrado en ISAF.

ISAF: Fuerza Internacional de Asistencia a la Seguridad.

LZ: Zona de toma de helicóptero.

MEDCAP: Proyecto de asistencia médica a civiles.

OMLT: Equipos Operativos de Mentorización y Enlace.

OPI: Puesto de Observación e Información.

OTAN: Organización del Tratado del Atlántico Norte.

PSB: Base de apoyo provincial.

QRF: Fuerza de Reacción Rápida.

RCW: Mando regional oeste.

TN: Territorio Nacional

TOM: Transporte Oruga de Montaña.

ZO: Zona de operaciones. 